\title{
Article
}

\section{Bayesian Smoothing Splines}

\author{
Gary Venter *
}

* Columbia University; gv2112@columbia.edu; Tel.: +1-646-266-2495

\begin{abstract}
Fitting parameters on spline curves produces more parsimonious models, maintaining fit quality. Smoothing the splines reduces predictive variance. Individual splines are often fit by type of variable, e.g., in age-period-cohort models. Linear and cubic splines are most common. Several smoothing criteria have been used for parameter curves, with cubic splines fit by constraining the integral of splines' squared-second derivatives popular recently. Constraining the sum of squared second differences for linear splines is analogous. Generally the degree of smoothing is selected using cross-validation. Known spline dummy-variable matrices allow regression estimation of splines, with smoothing done via constrained regression. Smoothing criteria based on sums of squares or absolute values of parameters, as in ridge regression or LASSO, improves predictive accuracy and produces splines similar to smoothing by second-derivative constraints. Variables with very low t-statistics represent points where curve-shapes barely change. Eliminating those variables leaves knots concentrated where spline shapes change. A Bayesian version of this puts shrinkage priors on spline parameters. This yields realistic joint parameter distributions, avoids problems associated with using cross-validation for parameter estimation, and readily expands to non-linear modeling, such as interactions among variable types. Regularized regression and Bayesian spline methods are compared for two example datasets.
\end{abstract}

Keywords: smoothing splines; shrinkage priors; MCMC; Bayesian methods

\section{Introduction}

Complex trends in data can be represented with curves pieced together across the data. Linear splines consist of line segments that join up at the points. Cubic splines use adjoining segments that agree in their first two derivatives, so are smoother, and tend to give more realistic interpolations near the joints (knots). Design matrices have been worked out to create these splines by regression.

Splines can also be fit across sets of enumerated variables in more general models. For instance, APC (age-period-cohort) models could have splines for each of the three sets of variables. The three spline design matrices would combine into one for the whole model. This can produce more parsimonious models. Variables are need for each A, P, and $\mathrm{C}$, but if adjacent parameters are similar, or lie on the existing curve, those spline parameters can be set to zero, as there is no change in the curve shape at those points.

Smoothing splines further increases model parsimony. Then the curves, and so the level parameters on the curves, do not swing as widely. This is a form of parameter shrinkage. Measures of curve smoothness are added to the negative loglikelihood (NLL), and are shrunk along with it . Before smoothing, the splines produce the same level parameters as would usual dummy matrices. Smoothing can improve predictive accuracy, although not fitting every value as closely. Here parameter-shrinkage methods like ridge regression, which are known to improve predictive accuracy, are investigated as smoothing 
criteria. Bayesian shrinkage using shrinkage priors is tried as well. This gives a wider range of smoothing choices.

Ridge regression, LASSO, and traditional spline smoothing cannot use likelihood penalized by parameter counts to compare models because shrunk parameters use up fewer degrees of freedom. Cross validation (CV) is used instead. The dataset is divided into a number of subsets, the NLL on each is calculated with the parameters estimated from the remaining data, and their sum is the CV NLL. It is thus an alternative method of penalizing the likelihood, and the degree of smoothing that optimizes the CV NLL is chosen. Estimating parameters by penalized likelihood has issues, however. The penalty is itself an estimate of sample bias - the overstatement of the likelihood due to measuring it on the sample used for fitting. The best penalized likelihood risks being the one with the greatest under-estimation of sample bias. From a Bayesian perspective, CV estimates are often close to the posterior mode. The Bayesian posterior mean of the shrinkage parameter comes from Bayesian shrinkage, and usually the mean is a better estimator than the mode, at least with regard to the estimation variance.

In a frequentist framework, parameters are constants that do not have distributions. But frequentists can use random effects, which are a lot like parameters with distributions. Their distributions are postulated, not subjective. But the same holds for priors in contemporary Bayesian analysis. They can be tested by model results just like other postulated distributions, like that of the data given the parameters. The main Bayesian engine is MCMC (Markov chain Monte Carlo) estimation, which samples from the joint likelihood of the parameters and the data. By the definition of conditional distribution this is the conditional distribution of the parameters given the data times the probability of the data. The latter is an unknown constant, and MCMC can sample without knowing it. In frequentist terms, it gives a sample of the conditional distribution of the random effects given the data. Being able to do this is largely a philosophical question for frequentists to decide. Some specifics are in the examples.

[1] give regression basis functions for cubic splines, with a derivation posted at [2]. Simplifying it a little, assume the spline has knots at the points $1: K$. They assume the spline is linear in $[1,2]$ and $[K-1, K]$. Dummy variables $a_{j}$ are defined for $j=1: K$. For an observation from any real $z \in[1, K]$ they set $a_{1}(z)=1$ and $a_{2}(z)=z$. Then for $j>2$ it works out that:

$$
\begin{gathered}
z>K-1: a_{j}(z)=(z+2-j)^{3} / K+2-j-(z+1-K)^{3} \\
z \leq K-1: a_{j}(z)=(z+2-j)_{+}^{3} / K+2-j
\end{gathered}
$$

[3] introduced a smoothing criterion for splines in general a century ago. The curve is differenced three times at each knot, and the sum of the third difference constrained. The integral of the squared second derivative of the curve gives a measure of its smoothness and is a popular criterion lately. With parameters $\beta_{j}$ the value of the cubic spline at any point is $\sum_{j} \beta_{j} a_{j}(z)$. The integral of the second derivative squared comes out to be $\left(\sum_{j} c_{j} \beta_{j}\right)^{2}$ for some values $c_{j}$. This is a weighted sum of all the cross products of the parameters. It generally increases simultaneously with $\sum \beta_{j}^{2}$ and with $\sum\left|\beta_{j}\right|$, so constraining either of those, as in ridge regression or LASSO, also constrains the squared second derivative, giving similar smoothing.

[4] provide a basis for linear splines. Again, $a_{1}(z)=1$. For $j, z>1, a_{j}(z)=$ $(1+z-j)_{+}$. This gives a design matrix for the second differences of the level parameters. 
Then constraining $\sum \beta_{j}^{2}$ constrains the sum of the squared second differences along the curve, analogously to the integral of the squared second derivatives of the cubic spline.

[5] introduced ridge regression, minimizing NLL $+\lambda \sum \beta_{j}^{2}$. They showed that there is always some $\lambda>0$ that gives a lower error variance than $\lambda=0$, which is the MLE (maximum likelihood estimation) case. MLE gives the minimum variance unbiased estimate. Ridge regression biases the estimates, generally by shrinking them towards the overall mean. Thus it ties in to Stein's Theorem ([6]), which says that with 3 or more means being estimated, the error variance is reduced by some degree of shrinkage towards the overall mean. With spline design matrices smoothing the splines generally improves the fit. There remains the issue of how much to shrink.

LASSO (least absolute shrinkage and selection operator), from [7], popularized by [8] instead minimizes NLL $+\lambda \sum\left|\beta_{j}\right|$. This is used more because it sets some parameters to exactly zero, thus taking them out of the model. That is the selection part. It still reduces the error variance by shrinkage towards the overall mean, verified for each model by cross validation for $\lambda$.

\section{Materials and Methods}

The first example looks at a sample of USA workers compensation benefit payments arranged by year of accident (rows = cohorts) by time to payment (columns = ages), as in Table 1. The years of payment (periods) are the upward sloping diagonals. The logs of the payments are fit by APC regression models.

Table 1. Workers compensation benefits paid by accident year and lag

\begin{tabular}{|c|c|c|c|c|c|c|c|c|c|c|c|c|c|c|}
\hline 129124 & 217397 & 118421 & 63984 & 39196 & 31450 & 19809 & 14556 & 8420 & 8507 & 7319 & 8937 & 10274 & 5383 & 5594 \\
\hline 216427 & 325705 & 171013 & 92336 & 65834 & 41287 & 28358 & 22541 & 15742 & 16934 & 12498 & 17058 & 10786 & 7834 & 0 \\
\hline 190698 & 207945 & 132229 & 74989 & 41989 & 32347 & 26640 & 21860 & 26870 & 17868 & 15149 & 15073 & 10549 & 0 & 0 \\
\hline 117415 & 166555 & 95082 & 55577 & 40785 & 28737 & 16396 & 15102 & 12993 & 16123 & 10323 & 8979 & 0 & 0 & 0 \\
\hline 133903 & 196230 & 110738 & 68289 & 52485 & 31817 & 23922 & 25356 & 17653 & 13431 & 14458 & 0 & 0 & 0 & 0 \\
\hline 201997 & 289239 & 165234 & 103281 & 70280 & 57042 & 39964 & 34252 & 25683 & 21589 & 0 & 0 & 0 & 0 & 0 \\
\hline 293284 & 435794 & 242665 & 159174 & 108122 & 75959 & 53191 & 42359 & 35453 & 0 & 0 & 0 & 0 & 0 & 0 \\
\hline 409026 & 605225 & 381820 & 235599 & 162399 & 113710 & 81298 & 70265 & 0 & 0 & 0 & 0 & 0 & 0 & 0 \\
\hline 514682 & 799168 & 437650 & 281391 & 203616 & 143531 & 109599 & 0 & 0 & 0 & 0 & 0 & 0 & 0 & 0 \\
\hline 674265 & 886002 & 512631 & 364672 & 263383 & 215784 & 0 & 0 & 0 & 0 & 0 & 0 & 0 & 0 & 0 \\
\hline 692986 & 905049 & 546113 & 396918 & 266963 & 0 & 0 & 0 & 0 & 0 & 0 & 0 & 0 & 0 & 0 \\
\hline 700094 & 929014 & 590184 & 366655 & 0 & 0 & 0 & 0 & 0 & 0 & 0 & 0 & 0 & 0 & 0 \\
\hline 627820 & 834302 & 517097 & 0 & 0 & 0 & 0 & 0 & 0 & 0 & 0 & 0 & 0 & 0 & 0 \\
\hline 449942 & 552715 & 0 & 0 & 0 & 0 & 0 & 0 & 0 & 0 & 0 & 0 & 0 & 0 & 0 \\
\hline 326810 & 0 & 0 & 0 & 0 & 0 & 0 & 0 & 0 & 0 & 0 & 0 & 0 & 0 & 0 \\
\hline
\end{tabular}

The second example is log mortality rates for Danish women found in the Human Mortality Database for ages 50 - 89 for years of death 1967 - 2016. The mortality rates are computed as the number of deaths divided by the applicable population. The data used is age by year, with cohort computed as year of death - age at death. These year:age cohorts approximate the year-of-birth cohorts. With 50 ages and 40 periods there are $40+50$ $-1=89$ cohorts, $1878-1966$. The variables for each direction are numbered consecutively $1,2,3 \ldots$.

The linear and cubic spline design matrices are built for each dataset. The logs of payments and of the mortality rates are put into column vectors, with parallel vectors used to track the age, period, and cohort of each observation. The first age, period, and 
cohort are left out for the linear splines, with a constant term included as a column of all 1 's. The three individual-dimension spline design matrices are combined into larger ones for the datasets, with the age, period, and cohort variables grouped in columns.

These combined design matrices are singular because of the overlap among the three directions. There is no apparent way to eliminate the overlap and get true effects for each direction. Even leaving out a direction does not help. Fitting an age-period model with no cohorts implicitly assumes that there are no cohort effects, and the parameters are those for this assumption. It is testable by reviewing residuals by cohort. For spline models, some variables will naturally be eliminated - those for ages, periods, and cohorts where the curve shape changes minimally. Leaving out such variables makes the design matrix invertible. In the first example, the age-cohort model was fit first, and the least-significant variables were taken out, and then the period variables were put in. For the second example, the cohorts were left out initially.

The variables to eliminate were identified by a LASSO fit on the spline design matrices, using the $\mathrm{R}$ package glmnet. The function cv.glmnet does a cross-validation exercise for $\lambda$, using their algorithms to find a range of values for $\lambda$. One output is \$lambda.min, which they identify as the smallest reasonable degree of shrinkage. The variables whose parameters it sets to zero were the ones eliminated. This was done again once the third direction was put back in, leaving a starting set of variables for the analysis. Some of those latter also went to zero. For the cubic splines, the first variable in each direction is the constant term. Only one of those is kept. The second variable gives the age, period, or cohort number for the direction. Because cohort = period - age, these variables are linearly dependent, and one must be left out. In these examples, that already happened at the first LASSO stage. Also, glmnet adds a constant that is not shrunk, so the constant term is left out of the design matrix for this step.

Ridge regression parameter estimates for a given $\lambda$ have a closed-form expression that simplifies cross validation and Bayesian analysis. With design matrix $x$ and data $y$, straight regression parameters are given by:

$$
\beta=\left(x^{\prime} x\right)^{-1} x^{\prime} y
$$

With $U$ parameters, let $\mathrm{J}$ be the $U x U$ identity matrix with the upper right element replaced by zero. Then the ridge-regression estimate is:

$$
\beta=\left(x^{\prime} x+\lambda J\right)^{-1} x^{\prime} y
$$

The sum of squared residuals is $\sum(y-x \beta)^{2}$. This computes quickly enough to easily do leave-one-out (loo) cross validation for $\lambda$, and this takes only a few lines of $\mathrm{R}$ code. Just loop through the observations, computing for each the squared residual, and so the loglikelihood, with the parameters fit without that observation. Standard R non-linear optimization software can quickly find the cross-validation $\lambda$ with the highest loo loglikelihood. This is done for each example using cubic and linear splines.

The implementation of MCMC is done with the Stan system, using the Rstan and CmdStanR packages. The code is run from $\mathrm{R}$ but coded in the Stan language, which is converted to $\mathrm{C}++$ and compiled. Mainly it takes in the data and has code for the prior distributions. Here the priors for the parameters $\beta$, but not the constant, were taken as mean-zero normals with parameter $s$. This is considered Bayesian ridge regression, as the NLL of the parameters is a constant $+\beta^{2} / 2 s^{2}$, so their sum is $\lambda \sum \beta_{j}^{2}, \lambda=1 / 2 s^{2}$. The residuals are postulated to be normal with standard deviation sig. Both $\log (s)$ and $\log (\operatorname{sig})$ are assumed to be uniform distributed in $[-6,6]$, and the constant is uniform on $\pm 1.8 \times 10^{308}$, which is the limit of double-precision numbers on most computers.

Bayesian LASSO puts double-exponential priors on the parameters. The NLL is then a constant $+|\beta| / 2 s^{2}$, so gives LASSO. It does not, however, set any parameters identically 
to zero over the entire sample. It does for some parameters in most samples, but this can vary across the samples, so the means are never exactly zero.

The double exponential distribution is similar to a Students t-distribution with six degrees of freedom. With matching scale parameters, they agree in variance and kurtosis, and so in all existing positive moments of that $t$-distribution, in that the odd moments are zero for both. The different shapes close to zero are due to differing moments of $1 / X$ for the positive half of the distributions. The normal is of course the limit of the $t$ as the degrees of freedom increases, and for degrees of freedom less than six, the $t$ gets more heavytailed. The case of two degrees of freedom is sometimes a good shrinkage prior. It has:

$$
\begin{gathered}
F(x)=0.5+0.5 x\left[2 s^{2}+x^{2}\right]^{-0.5} \\
f(x)=\left(2+[x / s]^{2}\right)^{-1.5} / s
\end{gathered}
$$

Then for each $\beta,-\log (f(\beta))=1.5 \log \left(2+[\beta / s]^{2}\right)+\log (s)$, so for a fixed s, the constrained estimation would minimize $\mathrm{NLL}+\sum_{j} \log \left(2+\left[\beta_{j} / s\right]^{2}\right)$. That would constrain larger parameters less than the sum of squares or absolute values does, so would allow greater relative deviation from zero for larger parameters. It also shrinks the smaller parameters more.

Intermediate between cross validation and fully Bayesian estimation of $\lambda$ is a hybrid constrained Bayesian estimation. This is Bayesian estimation of the loo cross-validation $\lambda$. It estimates $\lambda$ using the full spline design matrix, where the fitted value for each point for a given $\lambda$ is the ridge-regression fitted value excluding that point. The residuals are still taken to be normally distributed with standard deviation sig, which is the other parameter in the model. The $\beta$ parameters are an intermediate calculation.

This can run much faster and generally gets mean $\beta$ parameters close to those from the full Bayesian analysis, but with much lower standard deviations. It generates a distribution of $\lambda$, and often its mode is close to the cross-validation $\lambda$.

The full Bayesian estimation builds a sample distribution of all the parameter sets that could have generated the data. The hybrid method has just the parameter sets that are ridge-regression parameters from some $\lambda$. That would not be as useful for distributional projections from the model but would provide a look at the parameters and the distribution of $\lambda$.

Stan uses MCMC to create a sample of the conditional distribution of the parameters given the data. It also provides an estimate of the loo cross-validation loglikelihood for the whole sample using Pareto-smoothed importance sampling [9]. The entire distribution of $s$ would be provided for risk analysis. A point estimate could be selected as the mean $s$ from the conditional distribution. This avoids using cross-validation for parameter estimation. Also the cross-validation estimate of $s$, under some common assumptions, is more like the mode of the conditional distribution.

The distributions postulated for the $\beta$ parameters are also in the typical setup for random effects, but in that case the constant, $s$, and sig are parameters. There is no apparent reason that these three could not also be taken as random effects with the postulated distributions. With that step, MCMC could be used in a frequentist estimation.

\section{Results}

Table 2 shows the resulting $\lambda$ s and loo cross-validation sum of squared residuals (SSR) for linear and cubic splines for the two examples. 
Table 2. Ridge-regression loo cross-validation results

\begin{tabular}{ccccc}
\hline & \multicolumn{2}{c}{ Example 1 Linear, Cubic } & \multicolumn{2}{c}{ Example 2 Linear, Cubic } \\
\hline$\lambda$ & 0.342 & 0.004 & 25.36 & 0.202 \\
SSR & 2.163 & 3.138 & 8.046 & 11.21 \\
\hline
\end{tabular}

A higher $\lambda$ for a given model means that more shrinkage was applied. The linear models smoothed more and had better fits by loo cross-validation SSR than did the cubic models.

The variables remaining for each model after LASSO with MCMC-fitted parameters are given in Table 3. The variables are identified as a, y, or c for age, year, or cohort. In the linear splines, the first age, year, and cohort are left out for identifiability, and this is also done for the cubic splines as those variables are all constant terms.

The hybrid MCMC - cross-validation estimated $\lambda$ for Example 1, linear splines is 0.402 , with a mode of 0.291 . The cross-validation estimate of 0.342 is between these. The cubic-spline estimate is 0.0066 , with mode of 0.0048 , which is still higher than 0.0040 from cross-validation. The means are higher than the modes as is usual for positively skewed parameter distributions, and they were higher than the CV estimates. Calculating modes for sample distributions is a bit involved. The $\mathrm{R}$ modeest package was used here with the half-sample mode estimator. That finds the smallest interval with half the sample in it, then repeats for that half, etc.

For Example 2, the MCMC linear spline $\lambda$ is 22.0 with standard deviation 7.7 and mode 24.4 , compared to $\lambda$ of 25.4 from cross validation. The mode is close to the CV estimate, but now the distribution seems to be negatively skewed. The MCMC cubic-spline $\lambda$ is 0.0033 , which gives less shrinkage than the $\lambda=0.2$ from cross validation. The MCMC and hybrid estimations here followed some of the cohort variations in more detail than did cross validation - which had a better SSR than the hybrid did.

In both examples for both splines, the hybrid $\beta$ parameters are close to those from the full MCMC, and the resulting level parameters on the splines are difficult to distinguish in a graph. Naturally, though, the parameter standard deviations from the hybrid fit are much smaller, as the parameters are constrained to be ridge-regression results.

The loo penalized loglikelihood for the linear spline fit in Example 1 is 74.9, with a standard error of 11.9. For the cubic spline it is 52.0 with a standard error of 8.5 . They are about 2 standard errors apart, so the linear spline fit is probably but not definitively better.In Example 2, the linear spline loo is 2676 with a standard error of 39.3. The cubic loo is 2376.5 with standard error 42.2. The linear spline fit is somewhat better there as well.

There are splines fit to each of age, year, and cohort components for each example. The sum of these components plus the constant term gives the fitted value for a data point. Figure 1 graphs these six splines for the Workers Comp payment example, Example 1, for the MCMC fits. The other fits give splines that are visually indistinguishable from these. Even though the cross-validation estimation shrinks less, the splines are robust to small changes in $\lambda$. This makes the weakness of cross validation less important but of course MCMC gives better interval estimates.

In this example there are 15 intervals in each direction, so they work on one graph. The cubic splines are smoother. The linear splines show corners where the slope changes, but the parameter shrinkage keeps them reasonably smooth. The cubic and linear splines in each direction look like rotations of each other. These offset to give similar fitted values. 
Table 3. Variables left after LASSO with MCMC parameter estimates

\begin{tabular}{|c|c|c|c|c|c|c|c|c|c|}
\hline \multicolumn{2}{|c|}{ Ex. 1 Linear } & \multicolumn{2}{|c|}{ Ex. 1 Cubic } & \multicolumn{2}{|c|}{ Ex.2 Cohort Linear } & \multicolumn{2}{|c|}{ Ex.2 Cohort Cubic } & \multicolumn{2}{|c|}{ Ex. 2 All Other } \\
\hline $\mathrm{cn}$ & 11.87 & cn & 11.47 & c2 & -0.0056 & c3 & -0.0033 & Linear & \\
\hline y2 & 0.289 & y2 & 0.092 & c3 & -0.0060 & c9 & 0.02559 & $\mathrm{cn}$ & -5.1208 \\
\hline y4 & -0.098 & y6 & 0 & c30 & 0.0028 & c10 & -0.0773 & a2 & 0.0825 \\
\hline y6 & 0.074 & y8 & 0 & c31 & 0.0017 & c11 & -0.0199 & a10 & -0.0043 \\
\hline y8 & 0.036 & y9 & 0 & c32 & 0.0018 & c12 & 0.14383 & a11 & 0.0071 \\
\hline y9 & 0 & y10 & 0.013 & c33 & -0.0028 & c13 & 0.0283 & a12 & -0.0022 \\
\hline y10 & 0 & y11 & -0.025 & c34 & 0.0004 & c14 & -0.0339 & a19 & 0.0087 \\
\hline y11 & 0.061 & y14 & 0.008 & c35 & 0.0042 & c15 & -0.1202 & a25 & -0.0052 \\
\hline y14 & -0.107 & & & c36 & 0.0033 & c16 & -0.0177 & a26 & 0.0181 \\
\hline y15 & 0.071 & & & c37 & 0.0020 & c17 & 0.08452 & y30 & -0.0063 \\
\hline c2 & 0.119 & c2 & 0.415 & c38 & 0.0034 & c18 & 0.03676 & y31 & -0.0044 \\
\hline c3 & -0.484 & c3 & -1.848 & c50 & -0.0015 & c19 & -0.0374 & y34 & -0.008 \\
\hline c4 & -0.232 & c4 & 3.163 & c51 & 0.0026 & c20 & -0.0984 & & \\
\hline c5 & 0.507 & c6 & -1.769 & c52 & -0.0003 & c21 & 0.10715 & Cubic & \\
\hline c6 & 0.159 & c7 & 0 & c53 & 0.0029 & c24 & -0.0175 & $\mathrm{cn}$ & -5.16 \\
\hline c9 & -0.157 & c8 & 0.425 & c54 & -0.0026 & c40 & 0.08974 & a3 & 0.1285 \\
\hline c10 & -0.025 & c11 & 0 & c55 & -0.0069 & c41 & -0.1814 & a7 & -1.4956 \\
\hline c11 & -0.192 & c12 & 0.012 & c56 & -0.0220 & c42 & -0.0154 & a8 & 2.2623 \\
\hline c12 & -0.032 & & & c63 & 0.0004 & c43 & 0.25192 & a9 & -0.5971 \\
\hline c13 & -0.113 & & & c64 & 0.0120 & c44 & -0.2621 & a10 & -0.7382 \\
\hline c14 & -0.168 & & & c65 & 0.0073 & c45 & 0.09924 & a11 & 0.5747 \\
\hline a3 & -0.785 & a3 & -0.748 & c66 & 0.0019 & c46 & 0.24626 & a12 & -0.1344 \\
\hline a5 & 0.105 & a5 & 2.904 & c67 & -0.0031 & c47 & -0.1843 & y3 & -0.0002 \\
\hline a6 & 0.023 & a6 & -3.018 & c68 & -0.0053 & c48 & -0.4701 & & \\
\hline a8 & 0.13 & a7 & 0.916 & c69 & 0.0023 & c49 & 0.65241 & & \\
\hline a10 & 0.061 & a10 & -0.071 & & & c50 & -0.2266 & & \\
\hline a12 & 0.143 & & & & & & & & \\
\hline a13 & -0.188 & & & & & & & & \\
\hline a14 & -0.029 & & & & & & & & \\
\hline
\end{tabular}

The cubic splines are graphed with dots at intervals of 0.2 to show the smoothness of interpolation. They were fit at intervals of 1.0, or possibly even longer if some parameters went to zero. The jumps in the last interval are a bit misleading. The basis functions were set up to make the splines linear within the first and last intervals with the same slope, given by the coefficient of the second spline variable. That's what looks like a jump near the right side of the graphs. But only the ending point of the interval was used in the fitting. Visually those final points appear to continue the curves established in the prior intervals. It would probably not be difficult to devise a new spline basis that just continues the current spline without making it linear in the last interval. 


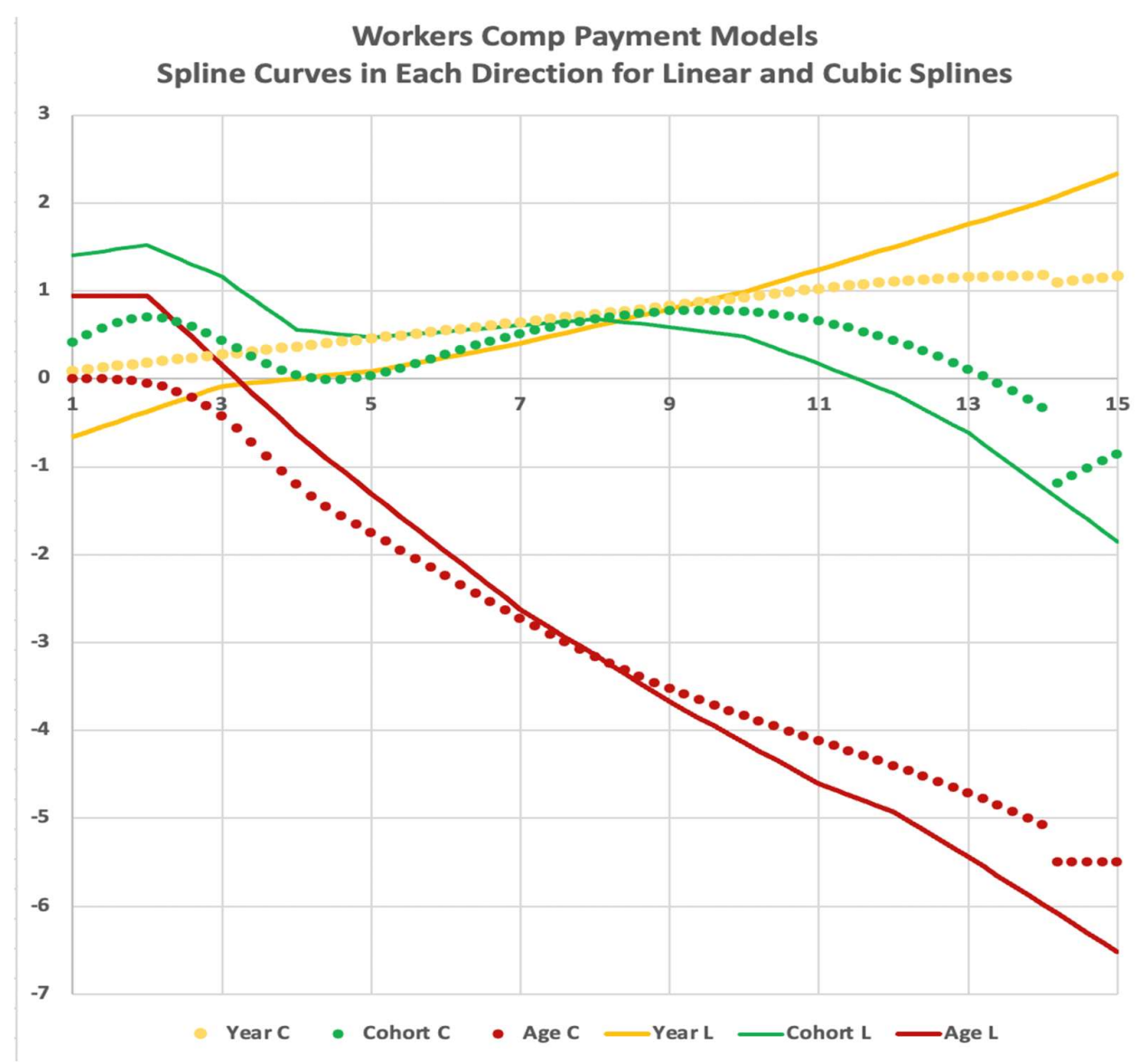

Figure 1. Spline curves Example 1, denoted as C or L for cubic or linear

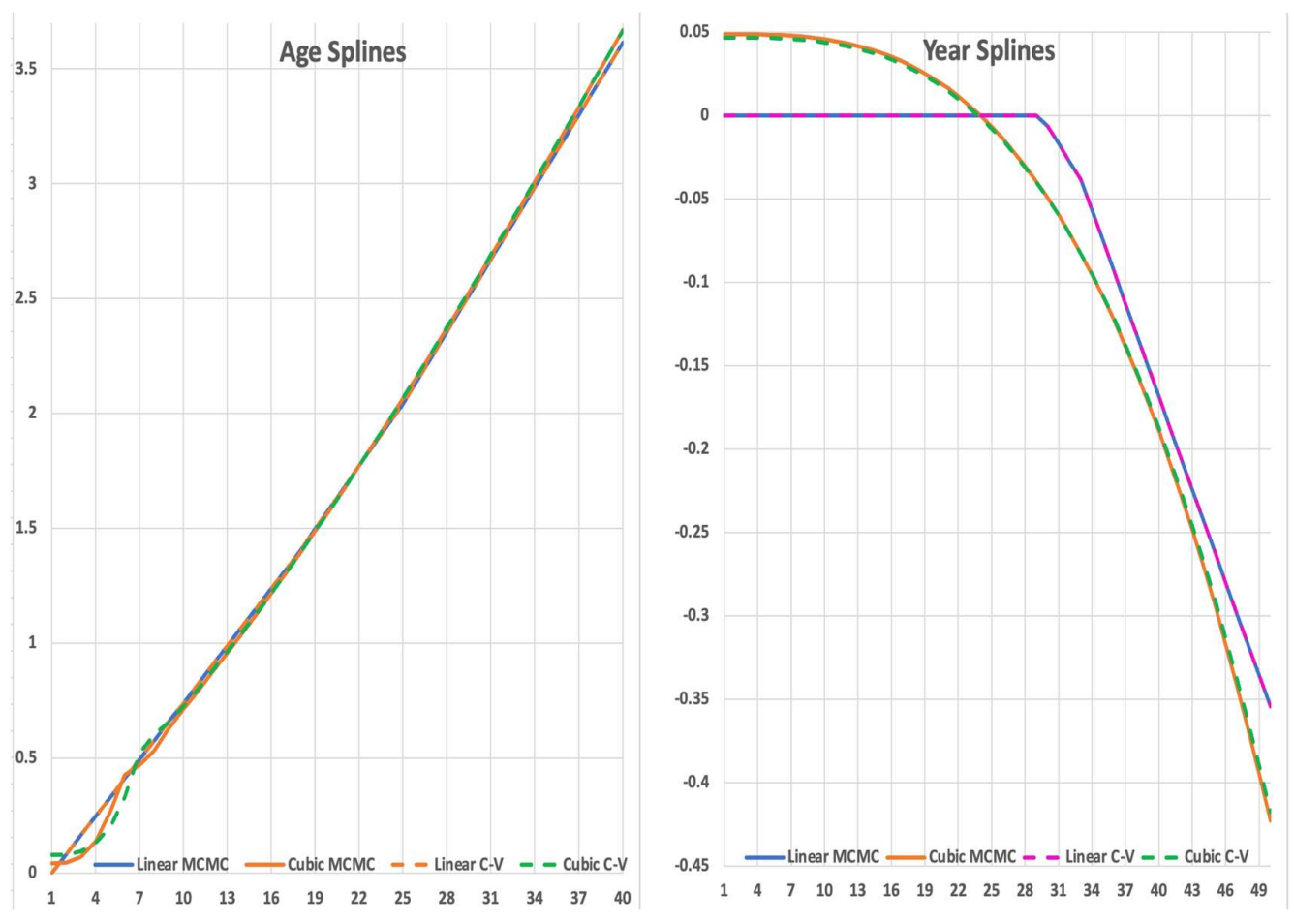

Figure 2. Age and year spline curves Example 2, denoted as MCMC or C-V. 
For Example 2, Danish female mortality, there are different numbers of points in each direction so separate graphs are used. Also the MCMC and cross-validation curves are slightly different, especially for cohort variabless for cubic splines. Figure 2 graphs the age and year splines and Figure 3 shows the cohort curves.



Figure 3. Cohort spline curves Example 2, denoted as MCMC or $\mathrm{C}-\mathrm{V}$ for cross validation.

The cross-validation and MCMC linear splines again look the same, and the cubic splines are smoother, but with more twists and turns. The cubic spline fit by MCMC was shrunk less and shows more detailed movements than the one fit by cross validation, especially for the cohort splines. The cross-validation cohort spline, where those details were smoothed over, was better.

In the linear spline fit, the cohort trend overall was almost double the time trend. This included a sharp increase starting for cohorts born in 1912, peaking around 1932, and not getting back to the previous trend until those born in the early 1940s. This is not a typical pattern. [9] and [10] studied Danish mortality drivers and found that smoking behavior is a cohort effect that explains much of the historical mortality pattern. Smoking tends to be a life-long habit, so would be a cohort effect. They found that there had been a strong increase in smoking among Danish women born between the two world wars, which is consistent with the model results here. 


\section{Discussion}

Using parameter shrinkage for spline smoothing results in reasonably smooth splines, and shrinkage methodology has known advantages, like reduction in error variances. LASSO software can be applied directly, and minimal LASSO shrinkage usually eliminates a few variables, and with them those knots, where the curve shape does not change much. The knots end up concentrated in areas where the shape changes the most.

Ridge-regression smoothing for a given $\lambda$ can be fit quickly with a single numeric calculation. This makes it fast and easy to use cross-validation for estimation of the smoothing constant $\lambda$. Bayesian shrinkage priors in MCMC give full distributions of the parameters and the resulting spline curves, and of $\lambda$ as well. They also avoid problems with cross-validation parameter estimation like understating sample bias and estimating by the mode. The overall fits to the data are not particularly sensitive to the degree of shrinkage, however, which mitigates the $\mathrm{CV}$ problems.

Linear splines had largely been abandoned for being too jagged, but spline smoothing helps with that. In these examples, the cubic spline curves are smoother, but the linear splines are not too jagged and give better fits. They may have more flexibility in matching the changes in curve shapes needed here. A hybrid using MCMC in cross validation averts some problems with CV estimation, and is faster than straight MCMC, but does not produce the full distribution of possible parameter sets. For one of the cubic splines, CV seems to give a more useful model than do the hybrid or Bayesian approaches.

Having simple formulas for spline smoothing enables modelers to put smoothing spline fitting into their own code for model development. MCMC does not require linear models, and spline parameter shrinkage can be included as needed in a wide variety of models. Some nonlinear extensions of Example 1 using linear splines are briefly covered next as illustration.

It is common to start with a model for logs of the data, and then use a log link for fitting to the original unlogged values. Fitting a gamma distribution with a log link is widely used in General Linear Models (GLM). The $j^{\text {th }}$ observation is postulated to be gamma distributed in $a_{j}, b_{j}$, which has mean and variance $a_{j} b_{j}, a_{j} b_{j}^{2}$. Usually some simplifying relationship among these parameters across the observations is assumed. In GLM the assumption is $a_{j}=a$, so this parameter is constant across the observations. Then the log link can give the $b_{j}$ parameters. The gamma's mean squared divided by its variance is $a$, so is then constant for all observations. Thus the variance is proportional to the square of the mean. But if $b$ is taken to be constant across the observations, then it gives the variance divided by the mean, which then is constant. In general, you could get the $b_{j}$ parameters from the $\log$ link and then for an estimated constant $c$, and any power $p$ you want, make the variance $=c \mu^{p}$ by setting:

Then:

$$
a_{j}=\left(c b_{j}^{p-2}\right)^{1 /(1-p)}
$$

$$
c\left(a_{j} b_{j}\right)^{p}=\left(c b_{j}^{-p}\right)^{1 /(1-p)}=a_{j} b_{j}^{2}
$$

Another popular generalization is to include interactions among the directions in the model. In mortality this started with the model of [11] that has the period trend modified by age. This is reasonable as medical improvements, etc., affect some ages more than others. Later [12] added cohorts to this model. The mean log mortality rate in year $i$ for age $j$ is:

$$
m_{i j}=c n+a_{j}+y_{i}^{b_{j}}+c_{i-j}
$$


This was used with linear splines in MCMC with a negative binomial fit and a log link by [13] and [14]. Like the gamma, there are two forms of the negative binomial that can be used, although GLM only uses one of them.

The model of [12] also works for Example 1. The period trends in workers compensation in the US are driven by medical cost inflation. The payments include wage replacement as well but that is usually not inflation adjusted. The wage portion also caps out in a few years for most workers. Medical becomes a growing part for later claim ages (which are the payment times since injury), and so the period trend affects later payments more.

The MCMC loo cross-validation loglikelihood is 74.9 for the APC log model above. This improves to 85.9 by including these interaction effects. Assuming a lognormal distribution for the actual losses using the log link produces all the same parameters but with a loo of -1266.6 . Likelihoods are lower for the unlogged values as the numbers are more spread out and the density is lower. For the gamma distribution with variance proportional to mean-squared, as in GLM, the loo is slightly worse, at -1268.3 . With variance proportional to mean, it is better, at -1257.7. Finally, using the $\mathrm{t}$-distribution with two degrees of freedom as the shrinkage prior brings this up to -1244.1, with a standard deviation of 13.6. Each step gives a possibly better result, and they add up to a meaningful improvement. The interaction term, choice of gamma distribution, and $t-2$ shrinkage prior are examples of the nonlinear modeling methods that can be built with spline models.

One limitation of Stan is that there are some probability distributions it cannot handle, including the Tweedie and the Poisson-Inverse Gaussian, which is a heavier-tailed negative binomial. These have good $R$ apps but those are specialized and Stan hasn't included them yet. The Poisson-Inverse Gaussian uses modified Bessel functions which move very slowly. It can take 40-50 digits of accuracy to compute their changes, and double-precision computers need special software for that. Stan cannot just call the R apps because it converts to $\mathrm{C}++$ and compiles. There are MCMC packages that can call anything from $\mathrm{R}$, but right now they are significantly slower.

The discussion here has emphasized what Bayesian shrinkage can do for smoothing splines, but the flip side of that is what smoothing splines can do for Bayesian shrinkage. Nearby parameters that are similar, or change in a regular way, can be put on splines that use fewer parameters. That can increase the parsimony and predictive accuracy of shrinkage models. Also it can help address the overlap problem of APC modeling. In APC models it is not always possible to eliminate variables with low t-statistics, as variables are needed in every position. Splines can deal with that in that omitted variables are just points where the curve shape doesn't change. The two methodologies work well in combination.

Data Availability Statement: The Danish mortality data is from the Human Mortality Database, University of California, Berkeley (USA), Max Planck Institute for Demographic Research (Germany) and United Nations, https://www.mortality.org/. For Danish data, go to the country section of https://www.mortality.org/cgi-bin/hmd/hmd download.php. Download the zip file (free account needed) and then find Exposures_lexis.txt and Deaths_lexis.txt under STATS.

Conflicts of Interest: The author declares no conflict of interest.

\section{References}

1. Hastie, Trevor, Robert Tibshirani, and Jerome Friedman. The Elements of Statistical Learning, Corrected 12th Printing; Springer, 2017. Available online:

https://web.stanford.edu/ hastie/ElemStatLearn//printings/ESLII_print12.pdf. (accessed on 142 2022).

2. Why are the basis functions for natural cubic splines expressed as they are? Available online: https://stats.stackexchange.com/questions/172217/why-are-the-basis-functions-for-natural-cubic-splines-expressed-as-theyare-es . (accessed on 132 2022). 
3. Whittaker, E. W. On a New Method of Graduation. Proceedings of the Edinburgh Mathematical Society 1922, Volume 41 February, $63-75$.

4. Barnett, G. and Zehnwirth, B. Best estimates for reserves. Proceedings of the Casualty Actuarial Society 2000, LXXXVII(167), 245321.

5. Hoerl, Arthur E. and Robert W. Kennard. Ridge Regression: Biased Estimation for Nonorthogonal Problems. Technometrics 1970, 12 (1): 55-67. https://doi.org/10.1080/00401706.1970.10488634.

6. Stein, C. Inadmissibility of the Usual Estimator for the Mean of a Multivariate Normal Distribution. Contributions to the Theory of Statistics 1956, 197-206.

7. F. Santosa and W.W. Symes. Linear inversion of band-limited reflection seismograms. SIAM J. Sci. Stat. Comput. 1986, 7(4), 13071330.

8. Tibshirani, Robert. Regression Shrinkage and Selection via the Lasso. Journal of the Royal Statistical Society: Series B (Methodological) 1996, 58 (1): 267-88.

9. Vehtari, Aki, Andrew Gelman, and Jonah Gabry. Practical Bayesian model evaluation using leave-one-out cross-validation and WAIC. Statistics and computing 2017, 27.5, 1413-1432.

10. Christensen, Kaare, Michael Davidsen, Knud Juel, Laust Mortensen, Roland Rau, and James W Vaupel. The Divergent LifeExpectancy Trends in Denmark and Sweden - and Some Potential Explanations. In Crimmins, E. M., Preston, S. H., \& Cohen, Panel on Understanding Divergent Trends in Longevity in High-Income Countries Committee on Population Division of Behavioral and Social Sciences and Education. 2010. https://www.ncbi.nlm.nih.gov/books/NBK62592/pdf/Bookshelf_NBK62592.pdf.

11. Juel, K., J. Sorensen and H. Bronnum-Hansen. Risk Factors and Public Health in Denmark. Scandinavian Journal of Public Health (Supplement 1) 2008, 36, 112-227.

https://journals.sagepub.com/doi/pdf/10.1177/1403494800801101.

12. Lee, R. and L. Carter. Modeling and Forecasting U.S. Mortality. Journal of the American Statistical Association 1992, 87, $659-75$.

13. Renshaw, A. E. and Haberman, S. (2006). A cohort-based extension to the Lee-Carter model for mortality reduction factors. Insurance: Mathematics and Economics 2006 38(3), 556-570.

14. Gary Venter and Şule Şahin (2021) Semiparametric Regression for Dual Population Mortality. North American Actuarial Journal, Published online: 30 Jun 2021.

15. Venter, Gary. A Mortality Model for Pandemics and Other Contagion Events. In BT - Pandemics: Insurance and Social Protection; M. del C. Boado-Penas, J. Eisenberg, and Ş. Şahin Eds.; Springer International Publishing, 2022; pp. 75-94.

\section{Appendix A}

This appendix has selected $\mathrm{R}$ and Stan code used in the study. This one is for LASSO.

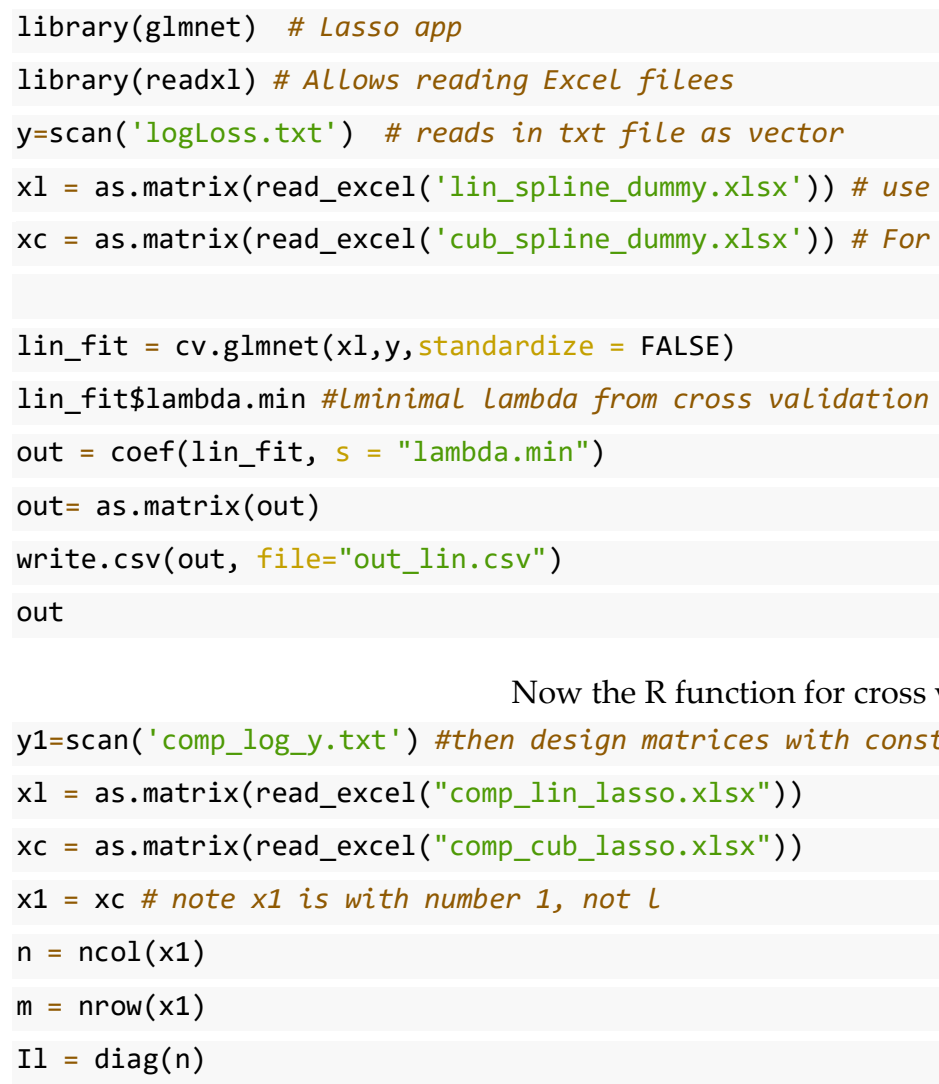

Now the $\mathrm{R}$ function for cross validation with ridge regression. 


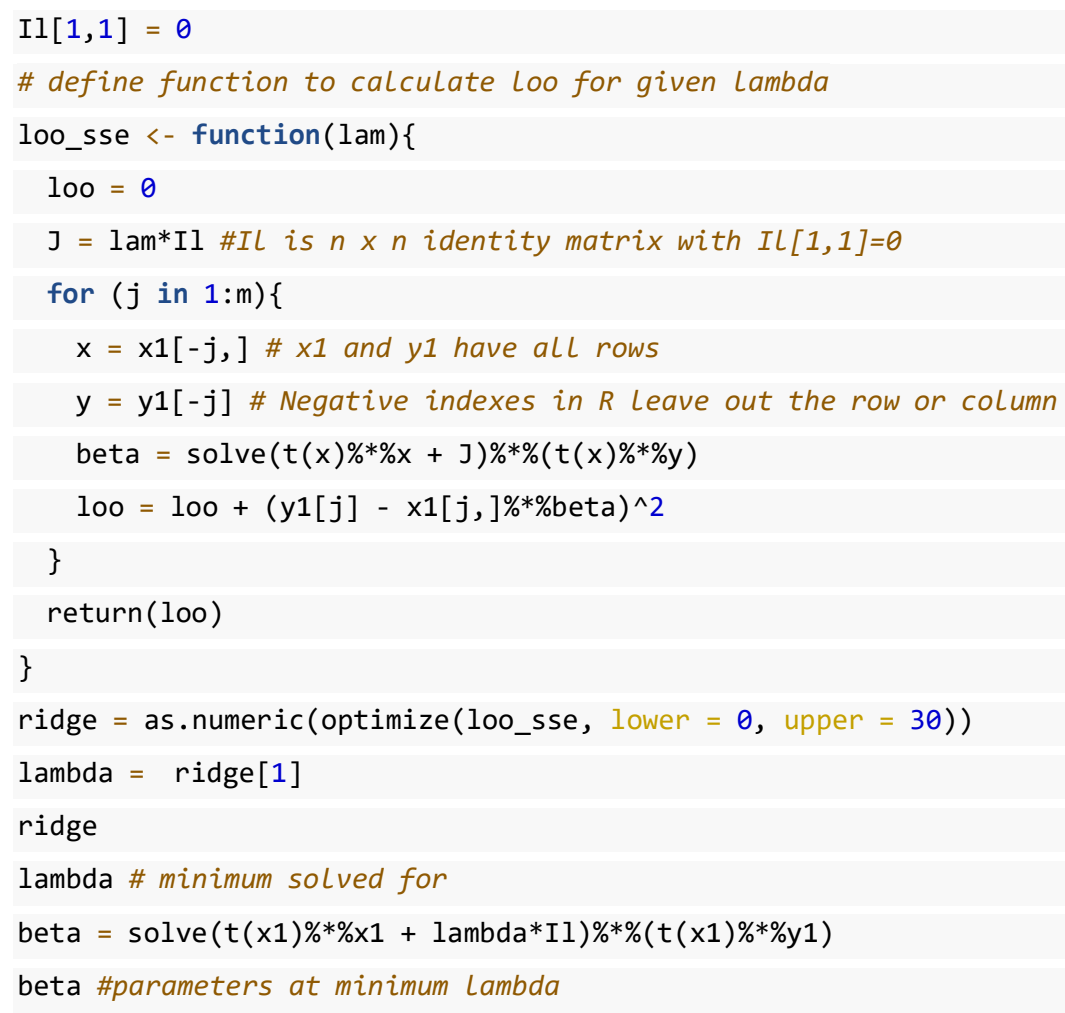

This is the R code to set up the Stan run for the hybrid CV-MCMC fit. It uses design library("10o") 


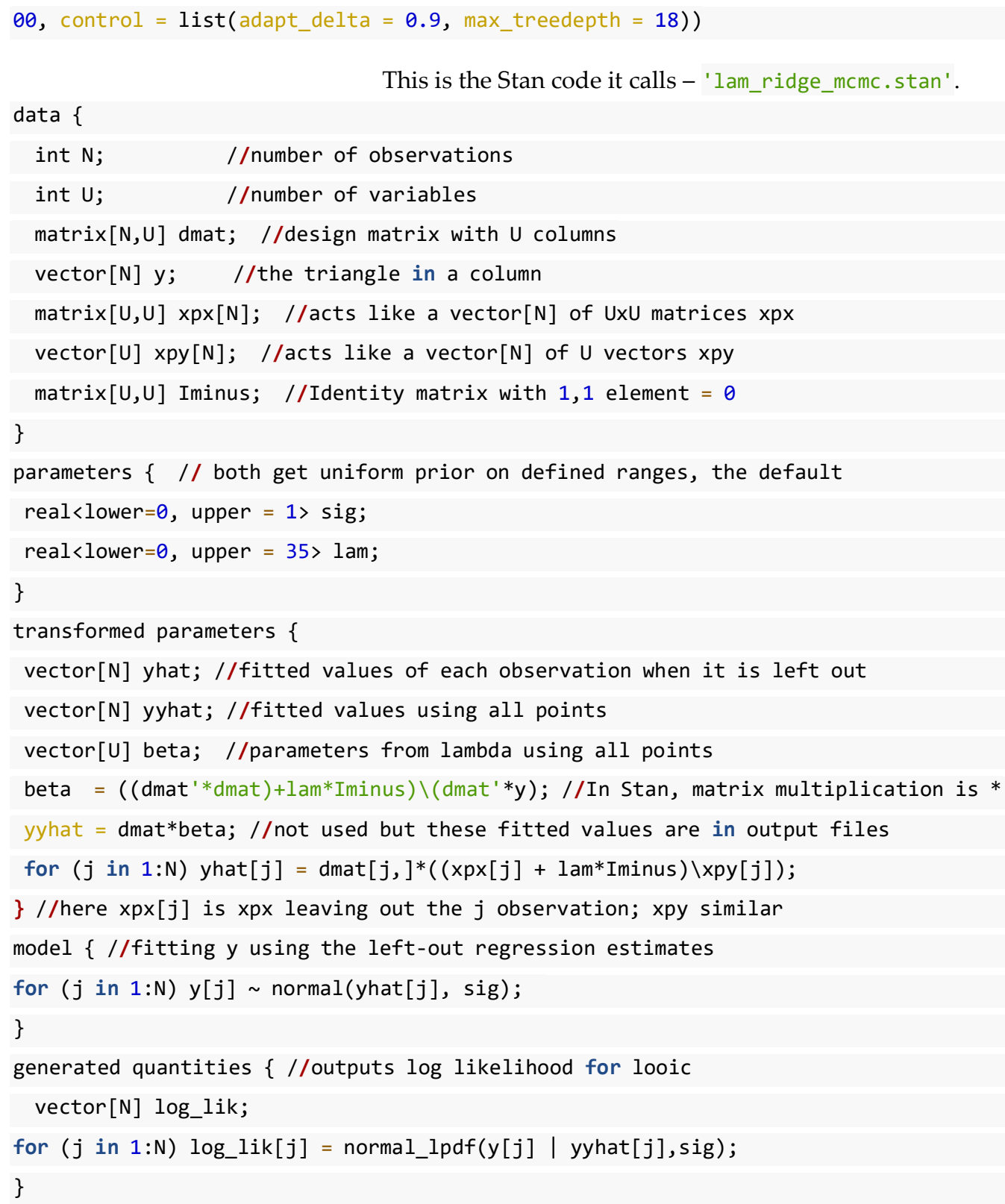

This is 'beta_ridge.stan' that it calls.

The straight MCMC run uses the design matrix without the constant term and calls the Stan code. 


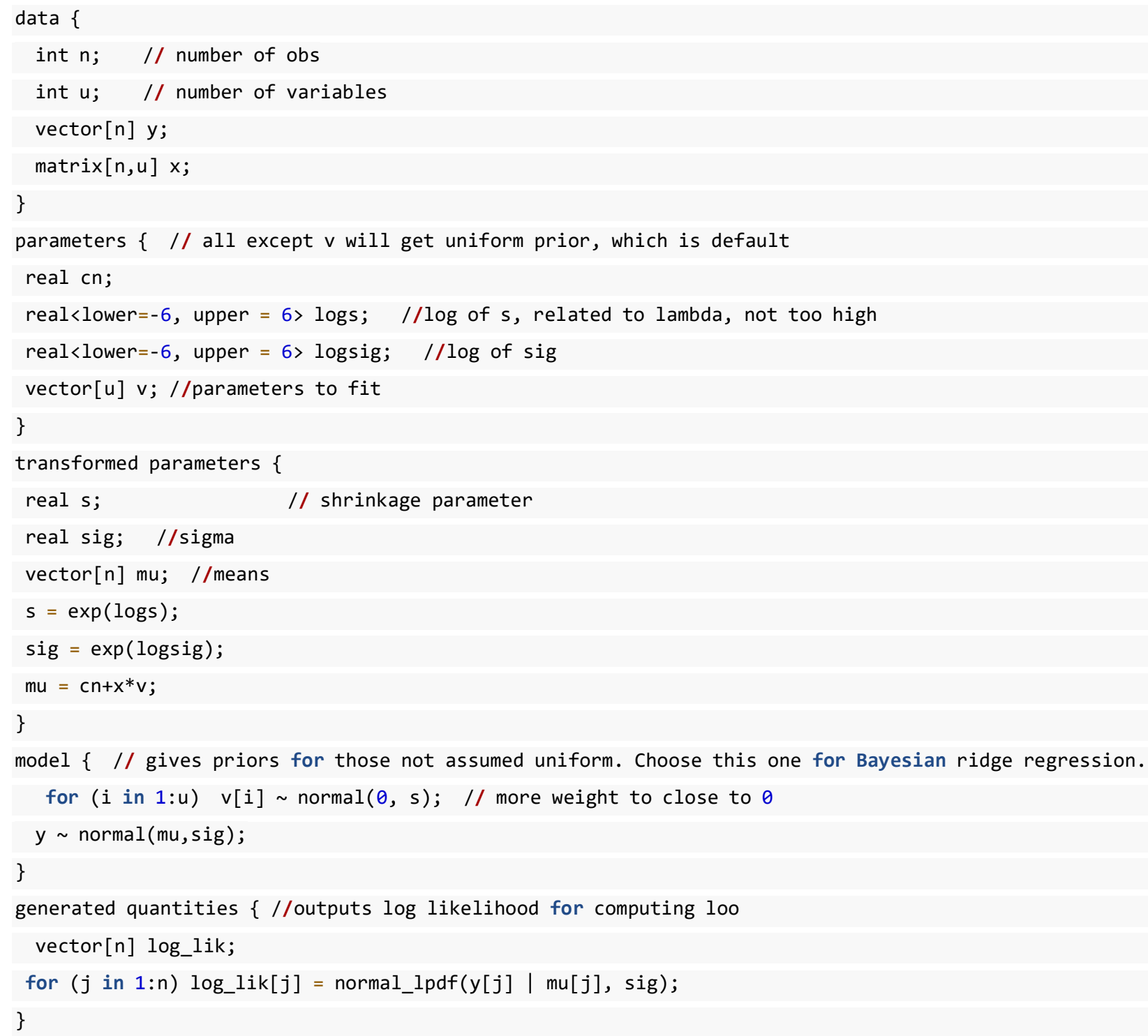

The code including an age-year interaction term starts with two design matrices one for cohorts and ages, in the rows and columns of the original dataset, and one for ages and year, the columns and diagonals. That one is later split into the two components, where the column factors are the trend weights applied to the year trends by age. The trend weights are standardized to be positive with a maximum at 1.0. The code here includes the log link and the gamma with variance proportional to mean. It is called lc for Lee-Carter.

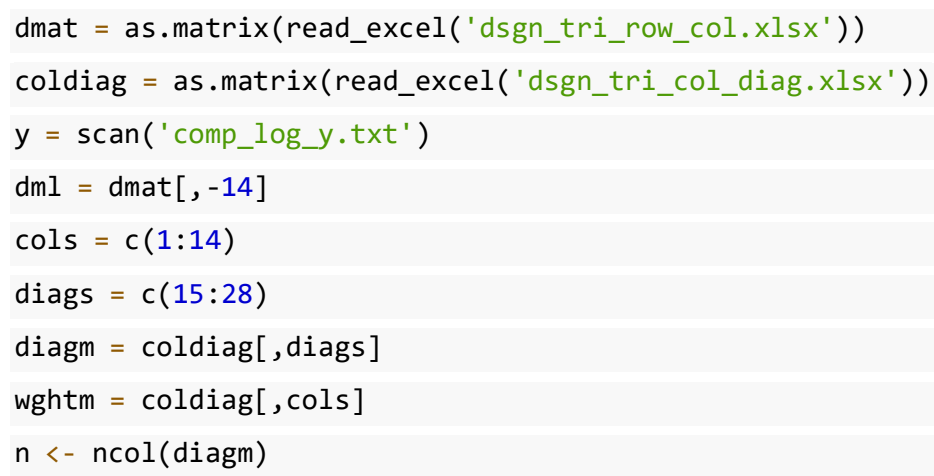




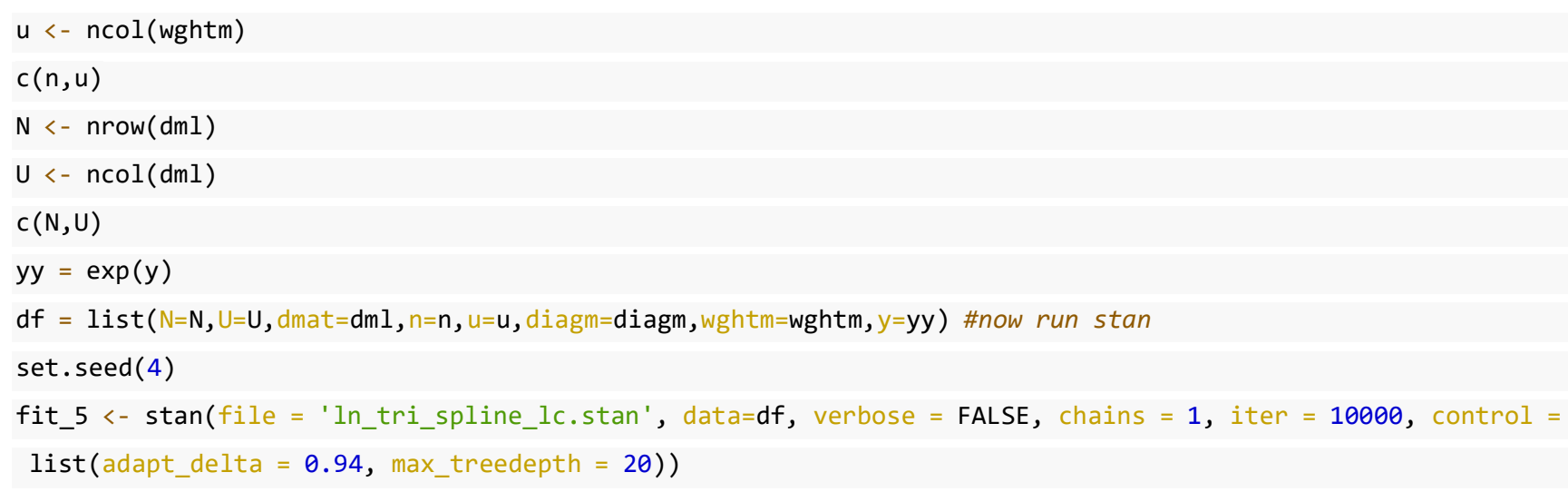

This is the Stan code 'In_tri_spline_lc.stan'.

data \{

int $\mathrm{N}$; $\quad$ //number of observations

int $U$; //number of variables

matrix $[\mathrm{N}, \mathrm{U}]$ dmat; //row col design matrix with $U$ columns

int $\mathrm{n}$; //number of diag params

int $u$; //number of wghts params

matrix $[\mathrm{N}, \mathrm{n}]$ diagm; //diag design matrix

matrix $[\mathrm{N}, \mathrm{U}]$ wghtm; //col wght dsgn mat

$\operatorname{vector}[\mathrm{N}] \mathrm{y}$; //the triangle listed in a column

\}

parameters $\{/ /$ all except $v$ will get uniform prior, which is default

real<lower $=-20$, upper $=20>1$ lncn; //ln constant term, starting in known range

vector [U] $\mathrm{v}$; //row-col parameters

vector $[\mathrm{u}] \mathrm{w}$; //trend-wghts parameters

vector $[\mathrm{n}] \mathrm{d}$; //diag parameters

real<lower $=-5$, upper $=5>\log s ; \quad / / \log$ of $s$, related to lambda, not too high

real $\langle$ lower $=-20$, upper $=20>$ logbeta; $/ / \log$ of gamma $b$ parameter

\}

transformed parameters \{

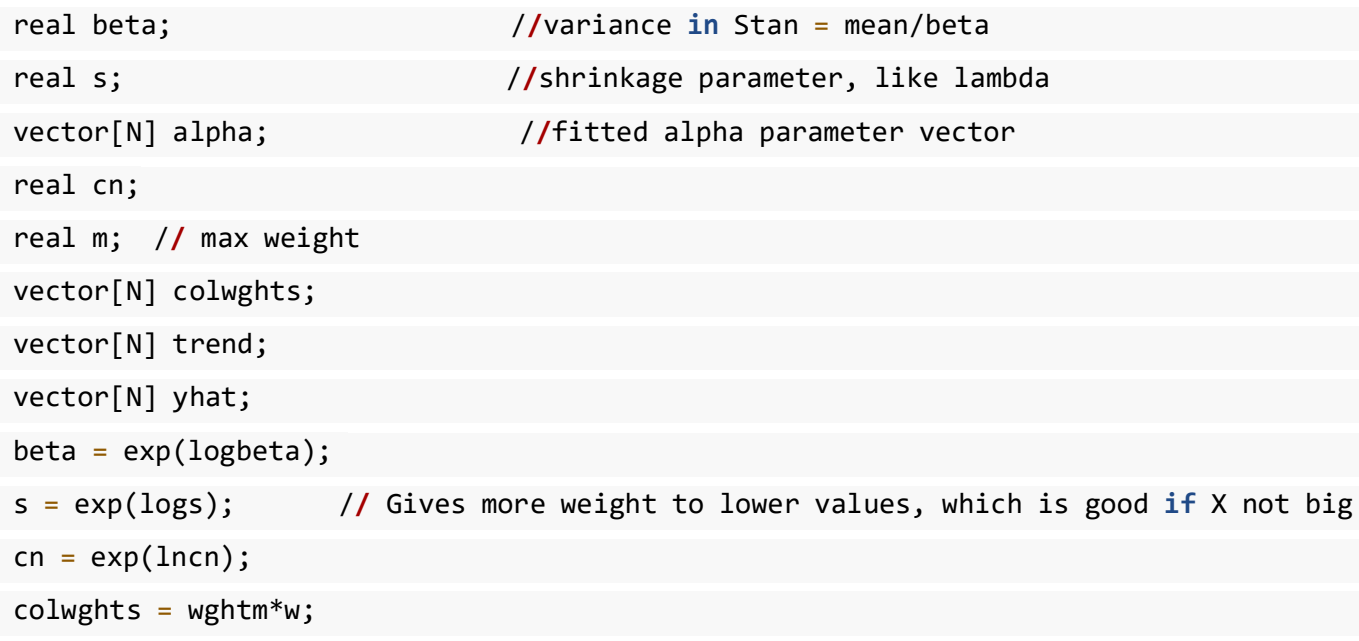






